\title{
A novel centralized beamforming scheme for radio-over-fiber systems with fixed wavelength allocation
}

\author{
Kota Ito ${ }^{\text {a) }}$ Mizuki Suga, Yushi Shirato, Naoki Kita, \\ and Takeshi Onizawa \\ NTT Access Network Service Systems Laboratories, NTT Corporation, \\ 1-1 Hikari-no-oka, Yokosuka-Shi, Kanagawa 239-0847, Japan \\ a)kouta.itou.te@hco.ntt.co.jp
}

Abstract: This paper proposes a novel centralized beamforming scheme for Radio-over-Fiber (RoF) systems with fixed wavelength allocation. The proposed scheme offers more wavelength utilization efficiency than the conventional beamforming methods that control wavelength allocation, and can control beam direction without explicit control by the base stations (BSs). Moreover, the scheme makes information about optical fiber length unnecessary, and supports systems that use high radio frequency (RF) signals or long optical fiber. Simulations evaluate and confirm the feasibility of the scheme for a system that uses $60 \mathrm{GHz}$ RF signals, $10 \mathrm{~km}$ optical fiber, and 8-element linear array antennas.

Keywords: radio-over-fiber, beamforming, millimeter-wave

Classification: Wireless Communication Technologies

\section{References}

[1] V. A. Thomas, M. El-Hajjar, and L. Hanzo, "Performance improvement and cost reduction techniques for radio over fiber communications," IEEE Commun. Surveys Tuts., vol. 17, no. 2, pp. 627-670, Second Quarter 2015. DOI:10.1109/ COMST.2015.2394911

[2] J. Beas, G. Castanon, I. Aldaya, A. Aragon-Zavala, and G. Campuzano, "Millimeter-wave frequency radio over fiber systems: A survey," IEEE Commun. Surveys Tuts., vol. 15, no. 4, pp. 1593-1619, Fourth Quarter 2013. DOI:10.1109/SURV.2013.013013.00135

[3] M. Oishi, H. Matsuno, K. Nishimura, and S. Akiba, "Experimental study of chromatic dispersion effects on antenna beam forming by RF over fiber," 2012 IEEE International Topical Meeting on Microwave Photonics, Noordwijk, pp. 140-143, 2012. DOI:10.1109/MWP.2012.6474076

[4] S. Akiba, M. Oishi, Y. Nishikawa, K. Minoguchi, J. Hirokawa, and M. Ando, "Photonic architecture for beam forming of RF phased array antenna," OFC 2014, San Francisco, CA, pp. 1-3, 2014. DOI:10.1364/OFC.2014.W2A.51

[5] ITU-T Recommendation G.694.1, Spectral grids for WDM applications: DWDM frequency grid, 2012.

[6] M. Tadokoro, T. Taniguchi, and N. Sakurai, "Optically-controlled beam forming technique for $60 \mathrm{GHz}-\mathrm{ROF}$ system using dispersion of optical fiber and 
DFWM," OFC/NFOEC 2007 - 2007 Conference on Optical Fiber Communication and the National Fiber Optic Engineers Conference, Anaheim, CA, pp. 1-3, 2007. DOI:10.1109/OFC.2007.4348909

[7] H. Huang, C. Zhang, C. Chen, T. Wu, H. Huang, and K. Qiu, "Optical true time delay pools based centralized beamforming control for wireless base stations phased-array antennas," J. Lightw. Technol., vol. 36, no. 17, pp. 3693-3699, Sept. 2018. DOI:10.1109/JLT.2018.2849001

[8] ITU-T Recommendation G.652, Characteristics of a single-mode optical fibre and cable, 2016.

\section{Introduction}

RoF is one of the solutions for future high-bandwidth wireless communications, because of its low attenuation, large bandwidth, and cost-effectiveness [1]. Millimeter-wave RoF systems are particularly attractive because the optical fiber provides long transmission, while the millimeter-wave signals provide large bandwidths in the wireless domain. Hence, RoF systems can offer extended coverage of millimeter-wave communication cost-effectively by connecting a number of simple BSs to a sophisticated central station (CS) [2].

Due to the high free-space propagation loss of millimeter-wave transmission, beamforming is very effective for raising performance. To fully utilize the costeffectiveness of RoF systems by allocating as many functions as possible to the CS, it is desirable that only CS controls the beam direction to keep each BS extremely simple.

A beamforming method for RoF systems that uses time delays caused by chromatic dispersion is reported in $[3,4]$. This conventional method controls the beam direction by controlling wavelength allocation. However, this conventional method has four problems. First, (i) wavelength utilization is not efficient. Because beam direction is tied to wavelength allocation, the number of wavelengths required is much greater than the number of antenna elements. Second, (ii) explicit $B S$ control is necessary. Controlling wavelengths means that changing the beam direction is realized by switching the wavelength corresponding to a certain antenna element. If not all wavelengths corresponding to a certain antenna element are included in a single channel of the BS de-multiplexer (DEMUX), the DEMUX must be tunable. Third, (iii) information of optical fiber length is necessary to determine the wavelength allocation. The wavelength allocation must be backcalculated from fiber length, dispersion parameter, and RF carrier frequency, but accurately estimation of fiber length is not easy. Finally, (iv) if the $R F$ carrier frequency is high or the fiber length is long, it may be impossible to the wavelength difference needed. For example, if we assume a fiber length of $10 \mathrm{~km}$, typical dispersion parameter of $17 \mathrm{ps} / \mathrm{km} / \mathrm{nm}$ in the $1550 \mathrm{~nm}$ band, and RF carrier frequency of $60 \mathrm{GHz}$, the wavelength tuning range is $0.05 \mathrm{~nm}$. Even with the $12.5 \mathrm{GHz}$ grid of dense wavelength division multiplexing (DWDM), the wavelength spacing is about $0.1 \mathrm{~nm}$ in the $1550 \mathrm{~nm}$ band [5].

Related to the conventional method, in [6], a technique to expand the wavelength spacing is proposed. This technique potentially overcomes problems of 


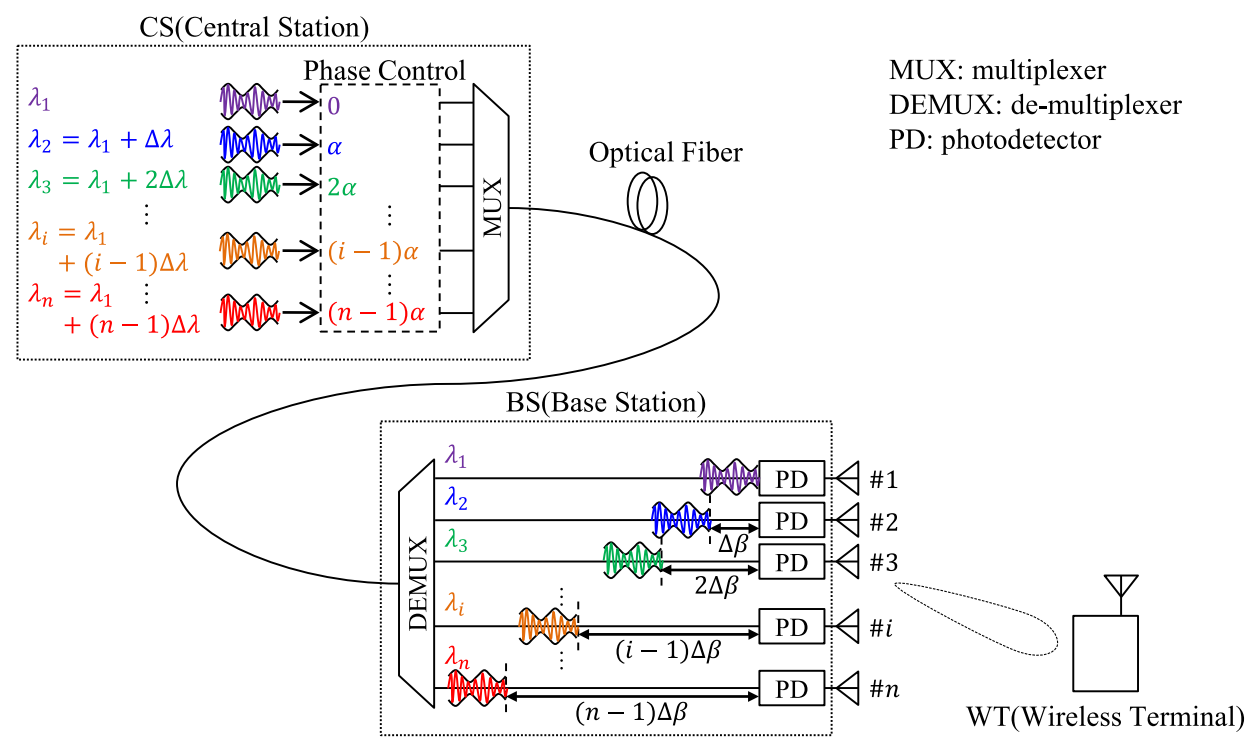

Fig. 1. Structure of proposed beamforming scheme.

(ii) and (iv), however, the modulation scheme of RF signals is restricted so that RF signals have several cycles like amplitude shift keying (ASK), and the other problems of (i) and (iii) remain. In [7], a centralized beamforming method without tunable DEMUX is proposed. Although this method can overcome problem (ii), BS structure is still complex and the other problems of (1), (iii) and (iv) remain.

This paper proposes a novel centralized beamforming scheme for RoF systems with fixed wavelength allocation to overcome the four problems (i)-(iv) detailed above. We evaluate the proposed scheme by simulations, and confirm its feasibility.

\section{Proposed beamforming scheme}

The structure of the proposed beamforming scheme is shown in Fig. 1. The scheme allocates fixed wavelengths with narrow and equal spacing, and sets the phases of signals to equal intervals at the CS. The RF phases of antenna elements are aligned with equal interval so that directed beams are formed.

The operation of the proposed scheme is as follows. First, to improve wavelength utilization efficiency, the BS directly connects wavelengths $\lambda_{1}, \lambda_{2}, \ldots, \lambda_{n}$ to antenna elements $1,2, \ldots, n$ respectively. The wavelengths $\lambda_{1}, \lambda_{2}, \ldots, \lambda_{n}$ are equally but narrowly spaced, yielding the representation

$$
\lambda_{i}=\lambda_{1}+(i-1) \Delta \lambda,
$$

where $\Delta \lambda$ is a small value.

Second, the optical signals are intensity modulated by an RF signal and phase controlled at CS. The RF phase of the modulated optical signal with $\lambda_{i}$ is adjusted to $(i-1) \alpha$, so RF phase differences between the modulated optical signals and adjacent wavelengths are $\alpha$. We should note that this phase control can be implemented on the RF signals before optical signal modulation. These phase controlled optical modulated signals are injected into the optical fiber, and transmitted to BS.

Third, the transmitted modulated optical signals are de-multiplexed and photodetected at the BS. Generally, the time delay difference between optical signals with 
$\lambda_{i}$ and $\lambda_{j}(1 \leq i<j \leq n)$ caused by chromatic dispersion with transmission through the fiber with dispersion parameter of $D(\lambda)$ and length $L$ is represented as

$$
\Delta \tau_{i j}=\int_{\lambda_{i}}^{\lambda_{j}} D(\lambda) \mathrm{d} \lambda \cdot L .
$$

Because the wavelength spacing $\Delta \lambda$ is narrow, we can assume that wavelengths $\lambda_{1}, \lambda_{2}, \ldots, \lambda_{n}$ have the same dispersion parameter, which can be represented as

$$
\exists D \in \mathbb{R}, \forall i, j ; D\left(\lambda_{i}\right)=D\left(\lambda_{j}\right)=D .
$$

The time delay difference $\Delta \tau_{i j}$ can be represented as

$$
\Delta \tau_{i j}=(j-i) \Delta \lambda D L .
$$

Hence the RF phase delay difference between the modulated optical signals with $\lambda_{i}$ and $\lambda_{j}$ can be represented as

$$
\Delta \beta_{i j}=\frac{2 \pi c \Delta \tau_{i j}}{\lambda_{R F}}
$$

In particular, the RF phase delay difference between the adjacent modulated optical signals with $\lambda_{i}$ and $\lambda_{i+1}$ is given by

$$
\Delta \beta_{i, i+1}=\frac{2 \pi c \Delta \lambda D L}{\lambda_{R F}}=\Delta \beta,
$$

as shown in Fig. 1. Then, the total RF phase difference between the adjacent modulated optical signals with $\lambda_{i}$ and $\lambda_{i+1}$ caused by the phase control at CS and the fiber transmission is $\alpha-\Delta \beta$, and the RF phase difference between the RF signals detected from the modulated optical signals with $\lambda_{i}$ and $\lambda_{i+1}$ is also $\alpha-\Delta \beta$.

Finally, the detected RF signals are transmitted from the antenna elements. From the above, the RF phase difference between the adjacent elements is given by $\alpha-\Delta \beta$, that is independent of antenna element number $i$. Hence the beam can be formed on direction $\theta$ which is defined by

$$
\frac{2 \pi d}{\lambda_{R F}} \sin \theta=\alpha-\Delta \beta
$$

where $d$ is the physical spacing of adjacent antenna elements and $\lambda_{R F}$ is RF signal wavelength.

Here, as we assume that fiber length $L$ is unknown, the relative phase delay difference $\Delta \beta$ is unknown, moreover the beam direction $\theta$ is also unknown. However, because the wavelengths $\lambda_{1}, \lambda_{2}, \ldots, \lambda_{n}$ are directly connected to different elements, the relative phase delay difference $\Delta \beta$ is constant, hence beam direction $\theta$ depends on just the value of $\alpha$, which can be set by the CS. Therefore, although the beam direction $\theta$ is unknown, it can be controlled by controlling the value of $\alpha$. To determine the beam direction, first beacons are transmitted from BS to the wireless terminal (WT) while scanning the value of $\alpha$. The feedback from the WT determines the value of $\alpha$.

We should note that in the proposed scheme the modulation scheme is not restricted unlike that of [6]. Furthermore, all that the BS has to do is just convert optical signals into electric signals and vice versa, so BS processing is yielding a simpler BS structure than that described in [7]. 
Table I. Simulation parameters

\begin{tabular}{l|l}
\hline RF Carrier Frequency & $60 \mathrm{GHz}$ \\
\hline Array Antenna at BS & 8 element linear array with half wavelength spacing \\
\hline Length of optical fiber & $10 \mathrm{~km}$ \\
\hline Dispersion Parameter & The upper limit value of ITU-T G.652.B [8] \\
\hline The First Wavelength $\lambda_{1}$ & $1524 \mathrm{~nm}$ \\
\hline Beam Direction $\theta$ & $0^{\circ}, 15^{\circ}, 30^{\circ}$ and $45^{\circ}$ \\
\hline
\end{tabular}

\section{Performance evaluation}

Since the proposed beamforming scheme assumes that the wavelength spacing $\Delta \lambda$ is narrow, we ran simulations to evaluate the beam patterns and determine the permissible wavelength spacing $\Delta \lambda$. By calculating the array factor (AF) of each wavelength spacing $\Delta \lambda$, we evaluated the maximum peaks and half-power angles of the AFs, which yielded the permissible wavelength spacing $\Delta \lambda$. The used parameters shown in Table I are based on an FWA system using the $60 \mathrm{GHz}$ unlicensed band. The fiber length is set to $10 \mathrm{~km}$, the typical length in optical access networks. The wavelengths are allocated in the manner of "point-to-point WDM overlay," $1524-1625 \mathrm{~nm}$.

Fig. 2(a), (b), (c) and (d) show the AFs normalized by the maximum peak of ideal $\mathrm{AF}$, for the beam directions of $0^{\circ}, 15^{\circ}, 30^{\circ}$ and $45^{\circ}$ respectively. For all directions, the main lobes decrease and the side lobes increase as the wavelength spacing widens, and a plurality of maximum peaks is confirmed if $\Delta \lambda \geq 1.44 \mathrm{~nm}$. The collapse of the beam pattern at large wavelength spacing is caused by the failure of the assumption that wavelengths $\lambda_{1}, \lambda_{2}, \ldots, \lambda_{8}$ have the same dispersion parameter.

Fig. 2(e) shows the maximum peaks of the normalized AFs, where $\Delta \lambda \leq$ $1.43 \mathrm{~nm}$, i.e. the beams have only one maximum peak. The maximum peaks of the normalized AFs decrease in the same way for all beam directions; the lowest is $-5.31 \mathrm{~dB}$ at $\Delta \lambda=1.43 \mathrm{~nm}$. Fig. 2(f) shows the half-power angles of the normalized AFs, where $\Delta \lambda \leq 1.43 \mathrm{~nm}$. For all directions, half-power angles increase sharply at $\Delta \lambda=1.31 \mathrm{~nm}$, because of the increase in side lobes.

Of particular note, in Fig. 2(e), the maximum peak of normalized AF is $-1 \mathrm{~dB}$ at $\Delta \lambda=0.96 \mathrm{~nm}$. Hence if the permissible peak attenuation relative to the ideal peak is $1 \mathrm{~dB}$, the wavelength spacing must be narrower than $0.96 \mathrm{~nm}$. Moreover, the half-power angles are not wide at $\Delta \lambda=0.96 \mathrm{~nm}$ as shown in Fig. 2(f). We should note that wavelength allocation with $0.96 \mathrm{~nm}$ spacing is not difficult, since the $100 \mathrm{GHz}$ grid of DWDM has the wavelength spacing with about $0.8 \mathrm{~nm}$ in the $1550 \mathrm{~nm}$ band. 


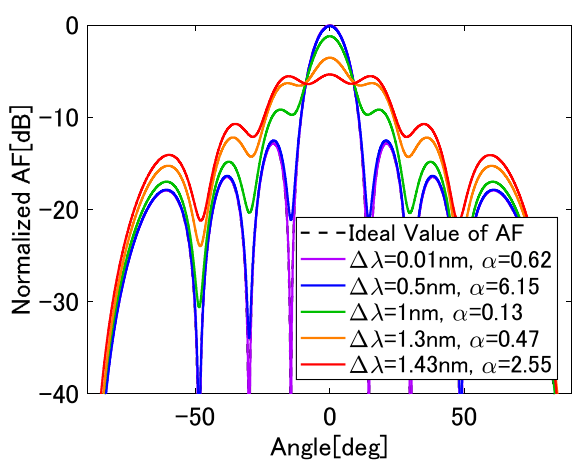

(a) Normalized AFs at $\theta=0^{\circ}$.

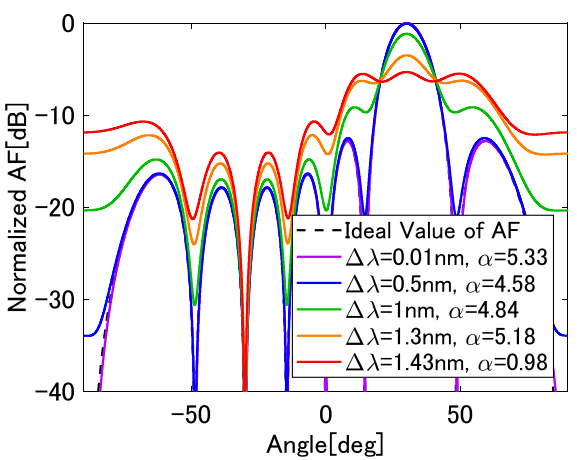

(c) Normalized AFs at $\theta=30^{\circ}$.

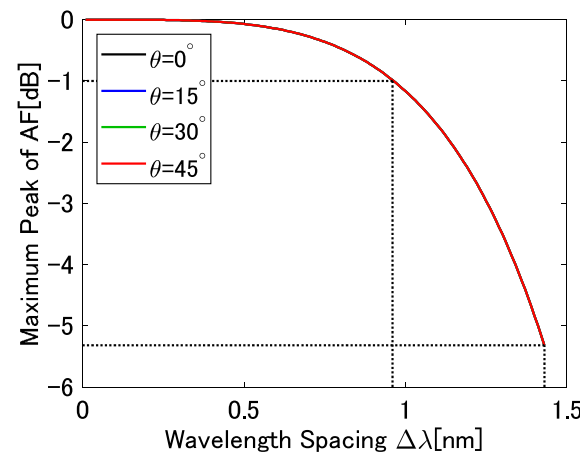

(e) Maximum peaks of normalized AFs.

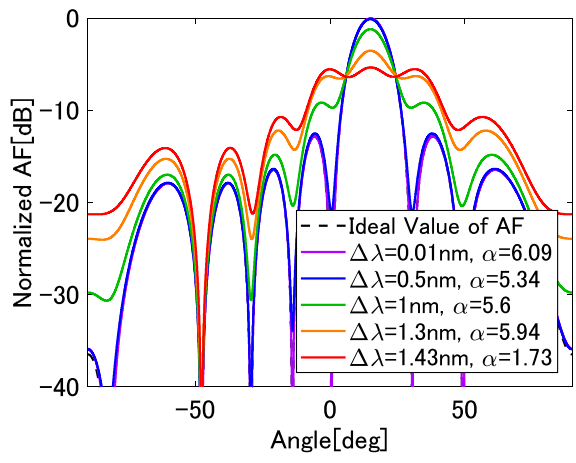

(b) Normalized AFs at $\theta=15^{\circ}$.

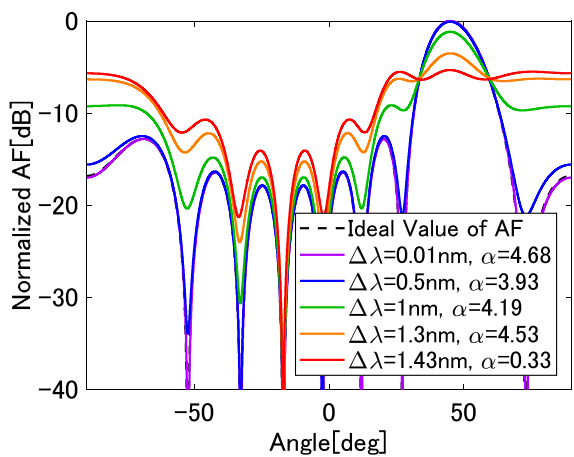

(d) Normalized AFs at $\theta=45^{\circ}$.

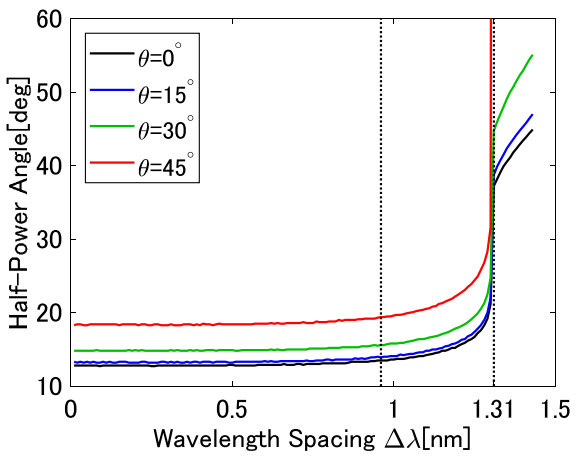

(f) Half-power angles of normalized AFs.

Fig. 2. Simulation results.

\section{Conclusion}

This paper proposed a novel centralized beamforming scheme for RoF systems with fixed wavelength allocation. The proposed scheme overcomes the problems of the conventional methods. The proposed scheme offers efficient wavelength utilization, and beam direction is controlled at the CS with no information of optical fiber length needed. In addition, simulations confirmed that the proposed scheme is applicable to FWA systems that use $60 \mathrm{GHz}$ RF signals and $10 \mathrm{~km}$ optical fiber, systems that the conventional methods fail to support. 